\title{
A Comparative Study of Force Measurements in Solution Using Micron and Nano Size Probe
}

\author{
Diganta Dutta $^{*}$, Roman Schmidt ${ }^{1}$, Samodha C. Fernando ${ }^{2}$, Indrani Ghosh Dastider ${ }^{3}$ \\ ${ }^{1}$ Department of Physics and Astronomy, University of Nebraska at Kearney, Kearney, NE, USA \\ ${ }^{2}$ Department of Animal Science, University of Nebraska-Lincoln, Lincoln, NE, USA \\ ${ }^{3} 1709$ West 38th Street, Kearney, NE-68845, Nebraska, USA \\ Email: *duttad2@unk.edu
}

How to cite this paper: Dutta, D. Schmidt, R., Dastider, I.G. and Fernando, S.C. (2019) A Comparative Study of Force Measurements in Solution Using Micron and Nano Size Probe. World Journal of Nano Science and Engineering, 9, 1-14. https://doi.org/10.4236/wjnse.2019.91001

Received: February 2, 2019

Accepted: March 26, 2019

Published: March 29, 2019

Copyright (๑) 2019 by author(s) and Scientific Research Publishing Inc. This work is licensed under the Creative Commons Attribution International License (CC BY 4.0).

http://creativecommons.org/licenses/by/4.0/

\begin{abstract}
Atomic force microscopy (AFM) is a device that is used for not only high-resolution imaging but also used for measuring forces. It is possible to quantify the surface density change for both colloid and nano probe as well as silica surface. By changing the quantity of ions within a potassium chloride solution, it then becomes possible to evaluate the quantity of ions that attach themselves to AFM colloid probe, nano probe and silica samples. In this study, the force was measured between AFM probes and silica surface in different ionic concentrations. Two different types of AFM probe were used: a colloid probe with a radius of 500 nano-meters and a nano probe with a radius of 10 nano-meters. This study is focused on measuring how the force magnitude, especially electrical double layer force, varied between the two types of probes by changing ionic concentrations. For all test trials, the results agreed with the electrical double layer theory. Although the micron probe was almost an exact match for all ranges, the nano probe was closest within its short-range forces. This is attributed to the formula use when analyzing the electrical double layer force. Because the formula was originally calculated for the micron probe, the shape and size of the nano probe created too many variables for an exact match. Along with quantifying the forces, this experiment allowed for an observation of Van der Waals force making it possible to calculate the Hamaker constant. Conclusively, all results show that the obtained surface charge density increases as the ionic concentration increases. In addition, through the comparison of the results obtained from the nano-sized probe and the micron-sized probe, it was concluded that nano size probe mapped higher surface charge density above the silica surface than the micron-sized probe under the same conditions.
\end{abstract}

\section{Keywords}

Atomic Force Microscopy, Surface Charge Density, Electrical Double Layer 


\section{Introduction}

In 1861 Thomas Graham first used the word "colloid" as a synonym to Adhesive [1]. Colloid systems have been used since the start of human civilizations [2]. One of the earliest examples is the colloidal pigment that was used for painting during the Stone Age. More modern examples include daily necessities like soap and cosmetics. One element of manipulating colloidal systems uses Interfacial forces, which are forces experienced between two bodies. To measure these forces, an instrument called the Surface Force Apparatus (SFA) has been used [3] [4] [5] [6] Using an SFA that had a 1-micron resolution, and a force sensitivity of 10-8 N Tabor et al. [3] conducted Van der Waals force measurements between two surfaces. Researchers have worked extensively to measure the surface forces between two microscopic samples by SFA before the invention of Atomic Force Microscopy [7]-[12]. With the advancement of cutting edge technology within the AFM, researchers are better able to measure Pico-Newton level forces [13]. AFM and SFA work similarly with the main difference being that the AFM measures force between the small probe and surface while SFA measures force between two macroscopic bodies. In addition to the development of the experimental techniques for measuring the interfacial forces, in 1941 Derjaguin-Landau and Verwey-Overbeek (DLVO) developed a theory for attractive Van der Waals forces and electrostatic forces between two samples [14]-[24]. Four basic interaction forces exist and they can be divided into two categories these categories are the short and long-range forces. Strong and weak forces act over short ranges, while electromagnetic and gravitational forces act over long ranges. The interfacial forces include the Van der Waals, electrostatic, hydration and steric forces. These forces are again divided into short-range and long-range forces. These forces are dominated when an AFM probe and a sample separation distance is about a nanometer. Inter-facial forces between the AFM probe and surface depend on the Hamaker constant, surface charge, and surface potential of materials. Electrostatic long-range forces were measured at different ionic concentrations. Short range Van der Waals forces were measured in DI water. Many researchers have used the unique AFM tool for interfacing force measurements between two surfaces. Larson et al. [11] measured interfacial force between titanium dioxide colloid and single crystal in an ionic solution. They measured interaction forces by changing $\mathrm{pH}$ values and ionic concentrations. Their experimental results show good agreement with theoretical Debye length. Olga et al. [25] measured forces between pairs of polystyrene particles in the electrolyte solution. They observed long range repulsive forces below $0.01 \mathrm{M} \mathrm{KCl}$ concentration. Their $0.001 \mathrm{M} \mathrm{KCl}$ concentration results show a good fit with the DLVO theory. Fielden et al. [26] measured interaction force between air bubbles 
and silica particles in an electrolyte. They observed repulsive forces at long ranges and attractive forces when bubbles and AFM tip distance decreases.

Meagher et al. [26] investigated hydrophobic interaction forces between a modified AFM tip and a polypropylene surface within a $\mathrm{NaCl}$ solution. They measured the attractive interaction force when the separation distances were about $30 \mathrm{~nm}$. Christenson et al. [27] measured attractive hydrophobic force between two mica surfaces in divalent solutions. In their experiments they used magnesium sulfate at different ionic concentrations and they also showed interaction magnitude decreased with increasing ionic concentration. Parker et al., [28] investigated force measurements between AFM tips and functionalize glass surface in a $\mathrm{NaCl}$ solution. They showed the magnitude of the force reduced with increasing salt concentration, but the attractive force magnitude increased. Kokkoli et al. [29] found that the strength of attractive force decreased when ethanol was added to water. They also showed a hydrophobic attractive force magnitude close to the Van der Waals force when the ethanol mole fraction increased about 75\%. Subir et al. [13] performed force measurements investigation between an AFM tip and a gold surface. Both surfaces are functionalized with bacteria. Their results showed agreement with extended DLVO theory.

Colloidal science, biomolecular transport, and drug delivery mostly used silica particles [28] [30]-[37]. One of the objectives of this study is to get a fundamental understanding of nano particles in a salt concentration at a very small scale. In this study, we quantify the force magnitude between micro and nano size probes against smooth flat surfaces under varying quantities of a salt solution. We also figured out the force trend in varying salt concentrations. In this study, we investigated how ions change in solutions with increasing salt concentrations. Our study shows that charge density increased with an increase of ionic concentrations.

\section{Method and Materials}

The sample surfaces were taken from a big silica wafer (Montco Silicon Technologies, San Jose, CA, USA) that was cut into $1.5 \mathrm{~cm} \times 1.5 \mathrm{~cm}$ squares. These silica surfaces were sonicated with a $1 \mathrm{M} \mathrm{KOH}$ (Acros Organics, New Jersey, USA) solution for about 15 minutes. Then these samples were rinsed with DI water for about 4 to 5 minutes. The sample surfaces were sonicated with acetone for about 15 minutes. After this step the samples were rinsed with acetone (Fisher Scientific, Pittsburgh, PA, USA) followed by DI water about 2 to $3 \mathrm{mi}-$ nutes. These sample surfaces were then sonicated with isopropanol (Fisher Science Education, Pittsburgh, PA, USA) for another 15 minutes; after this process, they were rinsed with isopropanol and DI water for 2 to 3 minutes. Finally, sample surfaces were sonicated in DI water following a 15-minute rinse with acetone, ethanol and DI water. These samples were dried by air gun and then put them above a hot plate at 120 degrees Celsius for 5 minutes. These sample surfaces were kept in clean boxes so that dust could not deposit above 
the sample surfaces.

\subsection{Van der Waals Force}

Van der Waals forces are the total of interaction forces between atoms and molecular dipoles of different particles [38]. An electromagnetic field is created when two particles approached each other less than $10 \mathrm{~nm}$ apart and electrons moved between particles while Van der Waals force is experienced. Van der Waals forces consist of three different kinds of forces such as Keesom force, Debey force and Dispersion force [39]. Dipole-dipole interactions between atoms or molecules are also known as Keesom forces. Another name for the Dispersion force is the London force. Dispersion forces are non-additive and bring atoms or molecules closer as well as align them. Dispersion force interacted between instantaneous dipole-induced dipole the most simplified approximation of the Van der Waals force between a sphere and a flat surface is [8]

$$
F_{v d w}=-\frac{A R}{6 h^{2}}
$$

In the above, $R$ is the radius of the sphere, $A$ is the Hamaker constant, and $h$ is the separation distance.

\subsection{Electrostatic Force}

Due to the ionization or dissociation process, solid surfaces become charged in aqueous solutions [40]. These ions on the silica surfaces are attracted by the equal and opposite charged of the ions in liquid and are distributed very close to the silica surfaces. Consequently, an electrical double layer form between the liquid and silica surface. The thickness of this double layer depends on the ionic concentration and varies from less than a nanometer to hundreds of nanometers. The AFM probe also becomes charged when it is immersed in liquid.

Electrostatic force occurs when the double layer of an AFM tip overlays with the double layer of the silica surface. The electrostatic force becomes repulsive when the surface charges of the AFM tip and silica surface are similar.

The corresponding Debye length for binary $z$. $-z$ electrolyte solution is

$$
\kappa^{2}=\frac{1}{\lambda_{D}^{2}}=\frac{2 \mathrm{e}^{2} z^{2} n_{\alpha}}{\varepsilon \varepsilon_{0} k_{B} T} .
$$

When the surface potentials or charge densities of the two objects are relatively low, Butt et al. [41] derived the electrical double layer force at constant potentials and surface charge densities,

$$
F_{E D L}=\frac{2 \pi R \varepsilon \varepsilon_{0}}{\lambda_{D}}\left[2 \Psi_{a} \Psi_{b} \mathrm{e}^{-h / \lambda_{D}}-\left(\Psi_{a}^{2}+\Psi_{b}^{2}\right) \mathrm{e}^{-2 h / \lambda_{D}}\right]
$$

And

$$
F_{E D L}=\frac{2 \pi R \lambda_{D}}{\varepsilon \varepsilon_{0}}\left[2 \sigma_{a} \sigma_{b} \mathrm{e}^{-h / \lambda_{D}}+\left(\sigma_{a}^{2}+\sigma_{b}^{2}\right) \mathrm{e}^{-2 h / \lambda_{D}}\right]
$$




\section{Results}

All AFM experiments were carried out by commercially available nanonics Multiview-4000 multi-probe AFM. For AFM image calibration, I have done standard silicon grid imaging. The AFM scanning images were taken in air at room temperature. The scanning speed was 30 micron per second. We had to wait for 30 minutes to stabilize the system. Before imaging the sample surface, I checked resonance frequency and quality factors of the AFM tip. The resonance frequency was $31,580 \mathrm{~Hz}$ and quality factor was 1058. And these values were matched with the manufacture's value. The AFM nano tip radius of curvature was $10 \mathrm{~nm}$. Before doing interfacial force measurements, we examined silica surfaces roughness. The AFM scanning area is $10 \times 10 \mu \mathrm{m}^{-2}$. The scanning speed was 20 microns per second. Figure 1 shows silica surface AFM topography image. The resolution of the AFM image is very high and its vertical resolution is less than $1 \mathrm{~nm}$.

\subsection{Force Measurements by Colloid Probe}

Between AFM colloid tip and silica surface in 0.0005 M KCL solution, interfacial forces were measured as shown in Figure 2. Experimental results along with theory fitted curves are given wherein the solid red line represents the theoretical results, and the solid blue line represents those of the experiment. The experimental curve shows a maximum force is about $13 \mathrm{nN}$, when the probe reaches the sample surface. The double layer thickness is about $13.56 \mathrm{~nm}$ at $0.0005 \mathrm{M}$ KCL solution, and hence, the Electrical Double Layer forces are experienced when the AFM tip and the substrate are $32 \mathrm{~nm}$ apart. Figure 2 shows repulsive forces due to the similarly charged surfaces and also osmotic pressure of the counter-ions. In $0.0005 \mathrm{M}$ KCL solution, force curve exponentially decreased with the distance of about $15-20 \mathrm{~nm}$. The experimental curve was fitted with theoretical results using Matlab programming. The AFM tip and silica surface densities as a result of the curve fit are given in Table 1 . The maximum tip surface charge density was $-0.014398812 \mathrm{C} / \mathrm{m}^{2}$ and the minimum was -0.012296685 $\mathrm{C} / \mathrm{m}^{2}$. The maximum silica surface charge density was $-0.002906252 \mathrm{C} / \mathrm{m}^{2}$ and the minimum value was $-0.0011996 \mathrm{C} / \mathrm{m}^{2}$. The average and $\mathrm{SD}$ curve fit results in tip and silica surface charge densities of $-0.01325( \pm 0.00106) \mathrm{C} / \mathrm{m}^{2}$ and $-0.00218( \pm 0.00088) \mathrm{C} / \mathrm{m}^{2}$, respectively.

Figure 3 demonstrates curves of force versus separation distance fitted with theoretical curves in different KCL concentrations with the use of a colloidal probe. The KCL concentrations given were 0.1, 0.01, 0.001 and $0.0005 \mathrm{M}$ wherein each condition, the experimental curves were matched with theoretical ones, and both agree. In higher concentration like 0.1 M KCL solution, the double layer thickness is $0.9 \mathrm{~nm}$; as a result, electro static forces were experienced at about $2 \mathrm{~nm}$ apart and had shown a lower magnitude of force. On the other hand, at a lower concentration like $0.0005 \mathrm{M}$, the KCL concentration had a higher magnitude of force. 


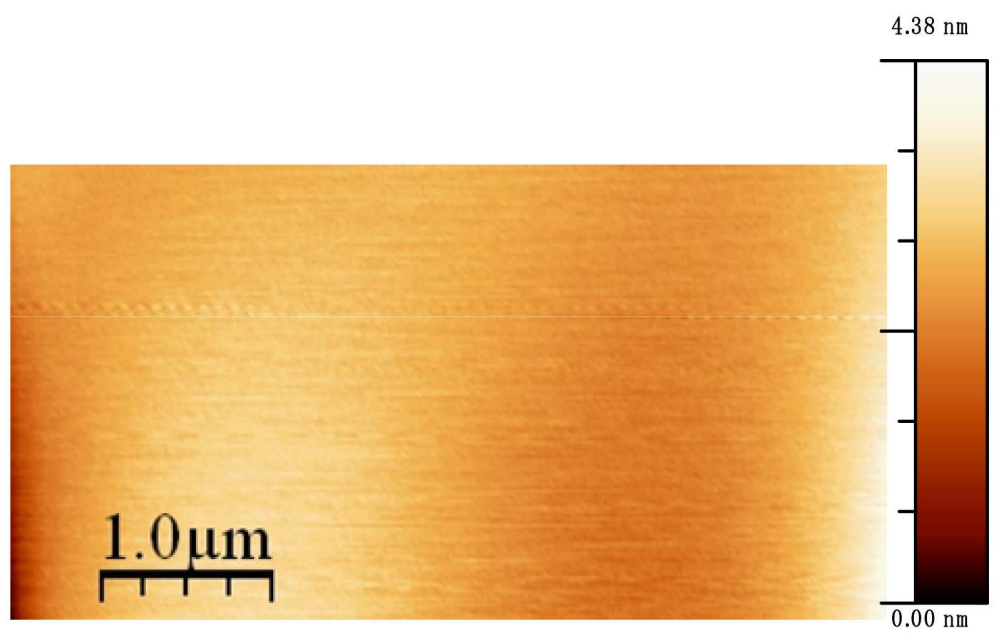

Figure 1. Silicon wafer $10 \times 10 \mu \mathrm{m}$ AFM topography image.

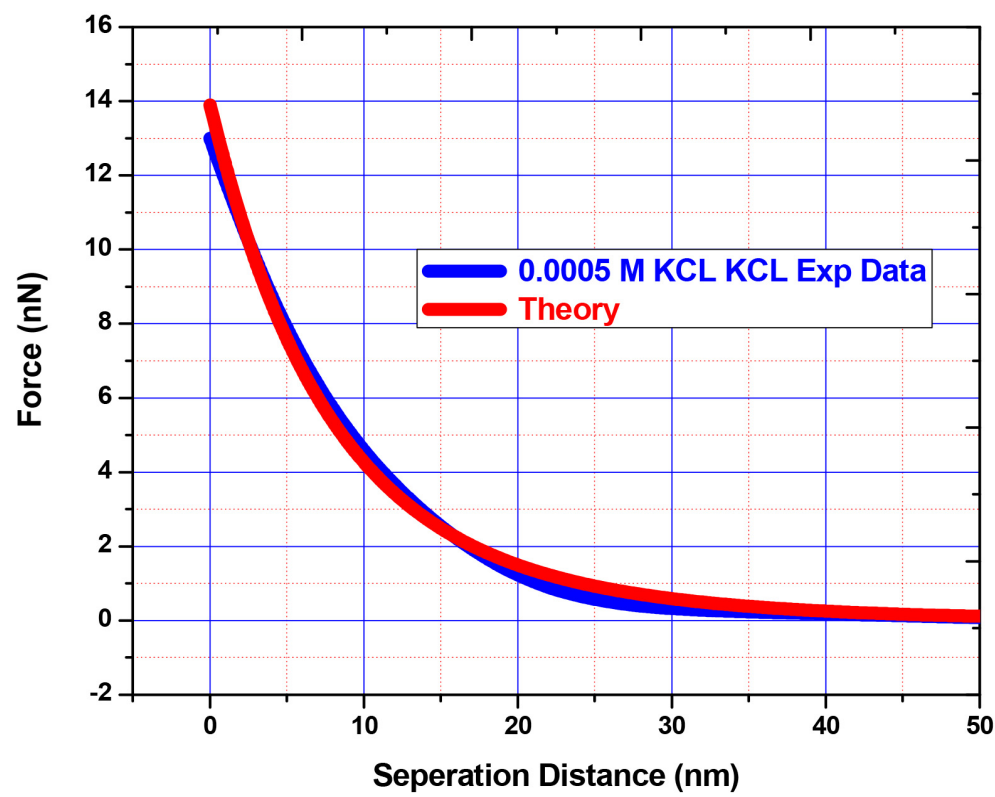

Figure 2. Force versus separation distance experimental curve fitted with theory in $0.0005 \mathrm{M}$ KCL solution using colloid probe.

Figure 4 is of the silica surface charge density versus a differential ionic concentration curve. The lower ionic concentration predicted a lower amount of charge density. In the $0.005 \mathrm{M} \mathrm{KCL}$ solution charge density is $-0.00218 \mathrm{C} / \mathrm{m}^{2}$. The charge density increased with increasing ionic concentration. At higher ionic concentrations, it exhibits a higher density whereas in the $0.1 \mathrm{M} \mathrm{KCL}$ concentration it shows that the silica surface density was $-0.01287 \mathrm{C} / \mathrm{m}^{2}$.

\subsection{Force Measurements Using Nano Probe}

Measured between the AFM's nano tip and silica surface in 0.0005 M KCL solution were interfacial forces. Figure 5 shows experimental results along with theory fitted curves. The solid red line represents the theoretical results, and the 
Table 1. Tip and Silica surface charge density in different experiments at $0.0005 \mathrm{M} \mathrm{KCL}$ solution.

\begin{tabular}{ccc}
\hline No of Experiments & $\begin{array}{c}\text { Tip Surface Charge Density } \\
\left(\mathrm{C} / \mathrm{m}^{2}\right)\end{array}$ & $\begin{array}{c}\text { Silica Surface Charge Density } \\
\left(\mathrm{C} / \mathrm{m}^{2}\right)\end{array}$ \\
\hline 1 & -0.013056099 & -0.002430071 \\
2 & -0.012296685 & -0.002906252 \\
3 & -0.014398812 & -0.0011996 \\
Avg. $( \pm \mathrm{SD})$ & $-0.01325( \pm 0.00106)$ & $-0.00218( \pm 0.00088)$ \\
\hline
\end{tabular}

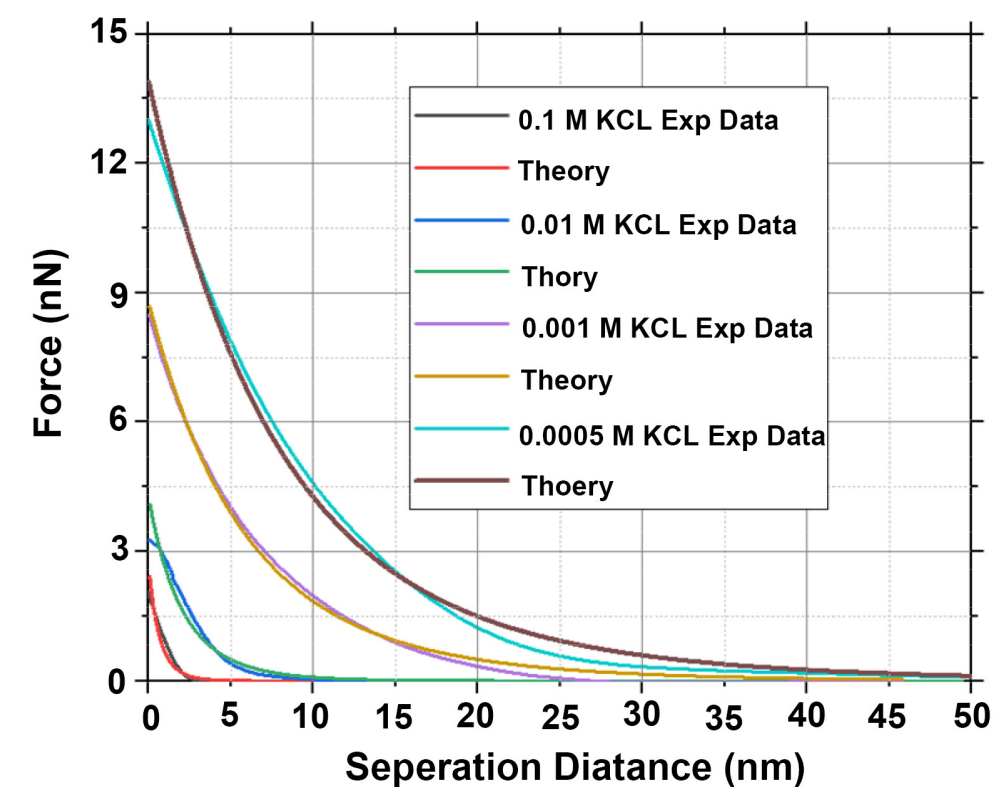

Figure 3. Four different KCL concentrations force versus separation distance experimental curve fitted with theory.

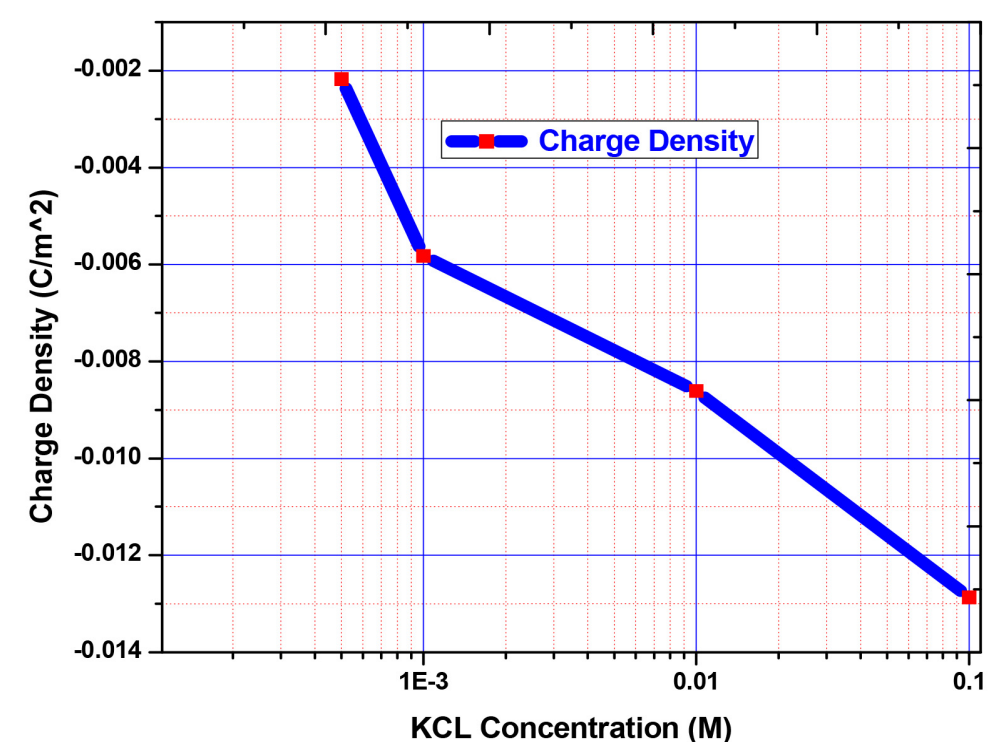

Figure 4. Charge density versus different KCL concentrations using colloid probe. 
solid blue line represents those of the experiment. The experimental curve shows a maximum force is about $4.5 \mathrm{nN}$, when the probe reaches to the sample surface. The double layer thickness is about $13.56 \mathrm{~nm}$ at $0.0005 \mathrm{M}$ KCL solution, and hence, the Electrical Double Layer forces are experienced when the AFM tip and the substrate are $32 \mathrm{~nm}$ apart. Figure 5 shows repulsive forces due to the similarly charged surfaces and also osmotic pressure of the counter ions. In $0.0005 \mathrm{M}$ KCL solution, force curve exponentially decreased with the distance of about 15 - $20 \mathrm{~nm}$. The experimental curve was fitted with theoretical results using Matlab programming. The AFM tip and silica surface densities as a result of the curve fit are given in Table 2 . The maximum tip surface charge density was -0.017313576 $\mathrm{C} / \mathrm{m}^{2}$ and the minimum was $-0.01575993 \mathrm{C} / \mathrm{m}^{2}$. The maximum silica surface charge density was $-0.003525419 \mathrm{C} / \mathrm{m}^{2}$ and the minimum value was -0.001587851 $\mathrm{C} / \mathrm{m}^{2}$. The average and SD curve fit results in tip and silica surface charge densities of $-0.01672( \pm 0.00084) \mathrm{C} / \mathrm{m}^{2}$ and $-0.00263( \pm 0.00098) \mathrm{C} / \mathrm{m}^{2}$, respectively.

Figure 6 shows force versus separation distance curves fitted with theory in different KCL concentrations using nano-probe as opposed to a colloidal probe. Experimental curves were fitted with electrical double layer theory. All of the experimental curves have good agreement with theory. At $0.01 \mathrm{M} \mathrm{KCL}$ solution, the double layer thickness is about $3.06 \mathrm{~nm}$ and the electrical double layer forces are experienced when the AFM tip and the substrate are $6 \mathrm{~nm}$ apart.

The experimental curve shows a maximum force is about $0.12 \mathrm{nN}$, when the probe reaches to the sample surface. At the lowest ionic concentration, the maximum force magnitude is about $4.5 \mathrm{nN}$.

Figure 7 shows silica surface charge density versus different ionic concentration curve. In the $0.0005 \mathrm{M} \mathrm{KCL}$ solution charge density is $-0.00263 \mathrm{C} / \mathrm{m}^{2}$. The

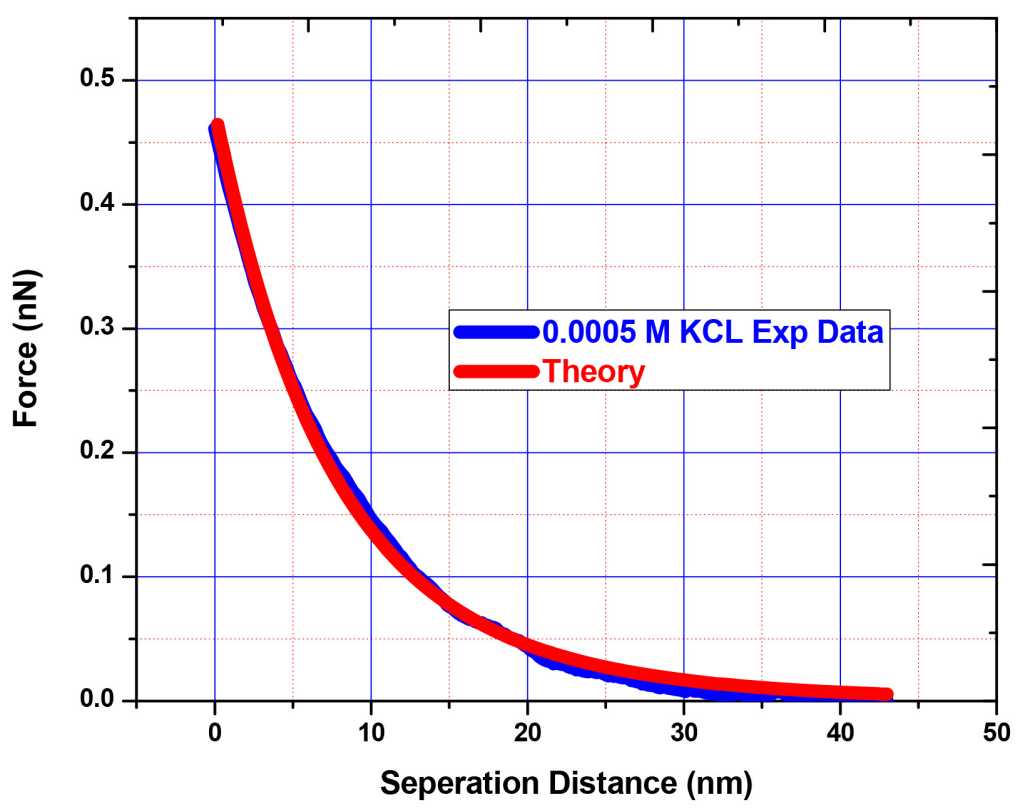

Figure 5. Force versus separation distance experimental curve fitted with theory in $0.0005 \mathrm{M} \mathrm{KCL}$ solution using nano probe. 


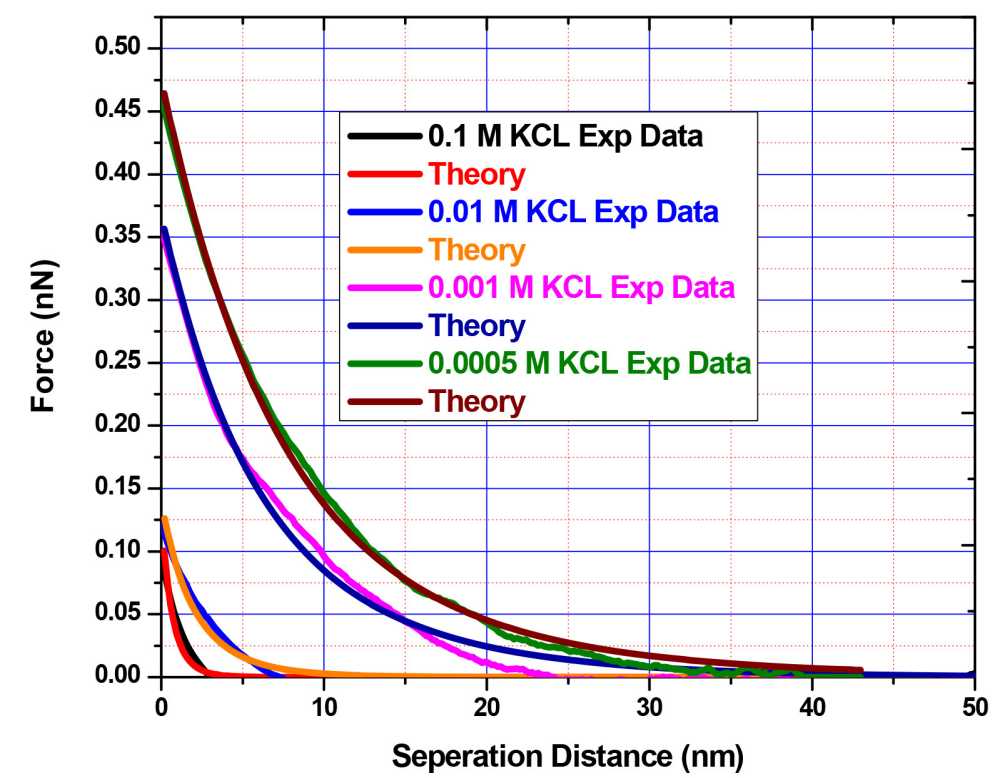

Figure 6. Force versus separation distance different experimental curves fitted with theory in different KCL solution using nano probe.

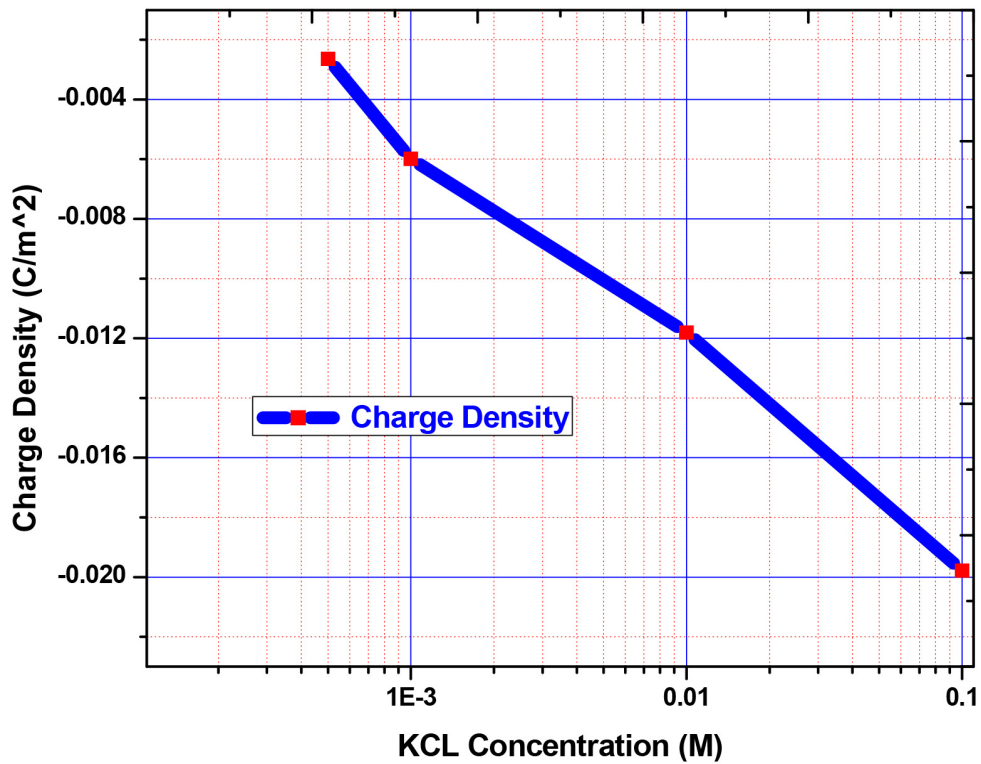

Figure 7. Charge density versus different KCL concentrations using nano probe.

Table 2. Tip and Silica surface charge density in different experiments at 0.0005M KCL solution.

\begin{tabular}{ccc}
\hline \multirow{2}{*}{ Experimental } & $\begin{array}{c}\text { Tip Surface Charge Density } \\
\left(\mathrm{C} / \mathrm{m}^{2}\right)\end{array}$ & $\begin{array}{c}\text { Silica Surface Charge Density } \\
\left(\mathrm{C} / \mathrm{m}^{2}\right)\end{array}$ \\
\hline 1 & -0.017078161 & -0.002777057 \\
2 & -0.017313576 & -0.001587851 \\
3 & -0.01575993 & -0.003525419 \\
Avg. $( \pm \mathrm{SD})$ & $-0.01672( \pm 0.00084)$ & $-0.00263( \pm 0.00098)$ \\
\hline
\end{tabular}


density increased with higher ionic concentration as well. 0.1 M KCL concentration shows silica surface density was $-0.01978 \mathrm{C} / \mathrm{m}^{2}$.

Figure 8 shows charge density measurements in different ionic concentration using different size of AFM probe. It also shows how charge density varies within ionic concentrations. Both the micron and nano-sized probe charge density increased with increasing of ionic concentrations. The qualitative increased of charge density with higher ionic concentrations agree with theoretical results got by Atalay et al. [40].

\subsection{Van der Waals Forces in DI Water}

AFM experiments were carried out in DI water by using the nano-sized AFM probe which radius of curvature is about $10 \mathrm{~nm}$ to measure the Van der Waals force. Figure 9 shows the force versus the separation distance in DI water, and attractive Van der Waals force is observed when the separation distance between the tip and the flat silica surface is very close. The measured force is fitted by the Van der Waals theory (symbols in Figure 9) to estimate the Hamaker constant. Based on the fit of the last part of the force curves, the estimated Hamaker constant is $\mathrm{A}=1.99 \times 10^{-19} \mathrm{~J}$. Hu et al. [42] investigated Van der Waals force measurements between silica and gold surface in DI water, and their Hamaker constant is $\mathrm{A}=1.6 \times 10^{-19} \mathrm{~J}$, which is close to our result.

\section{Conclusion}

It is feasible to map the surface charge density of silica surface and AFM collide probes as well as nano probe in $\mathrm{KCl}$ solution. To predict how the quantity of ions would affect the acquired force and the surface change density, electrostatic

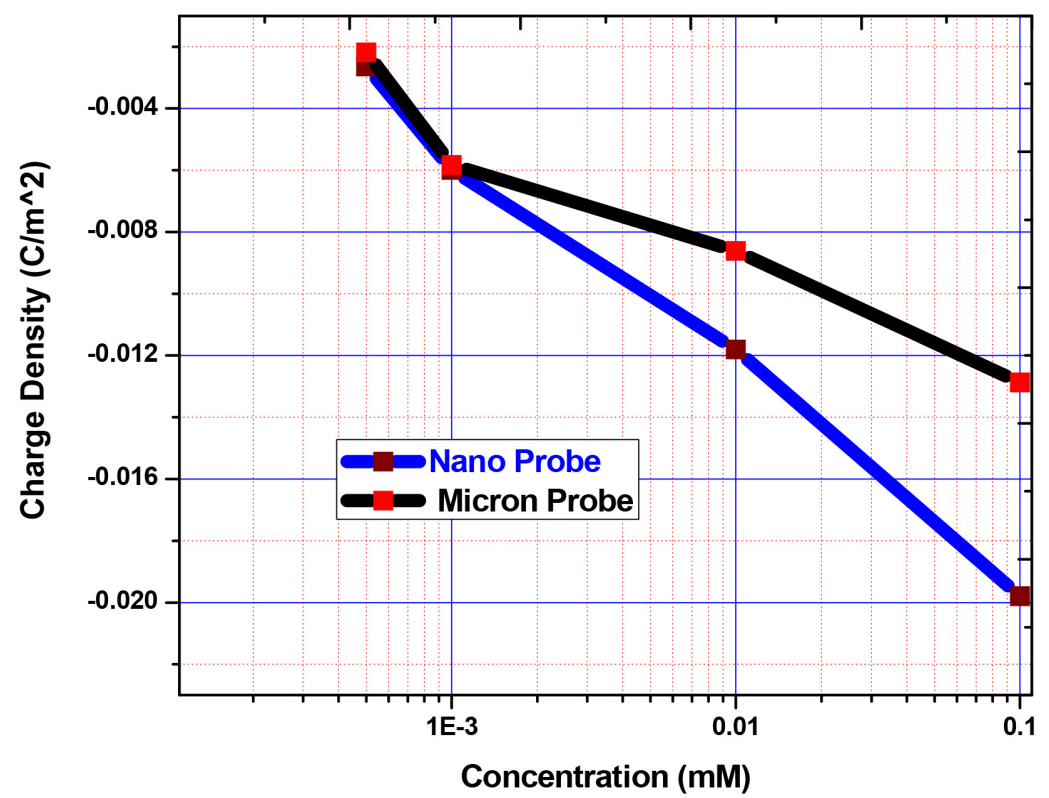

Figure 8. Comparison between the nano probe and micron probe surface charge density mapping above the silicon wafer in different ionic concentrations. 


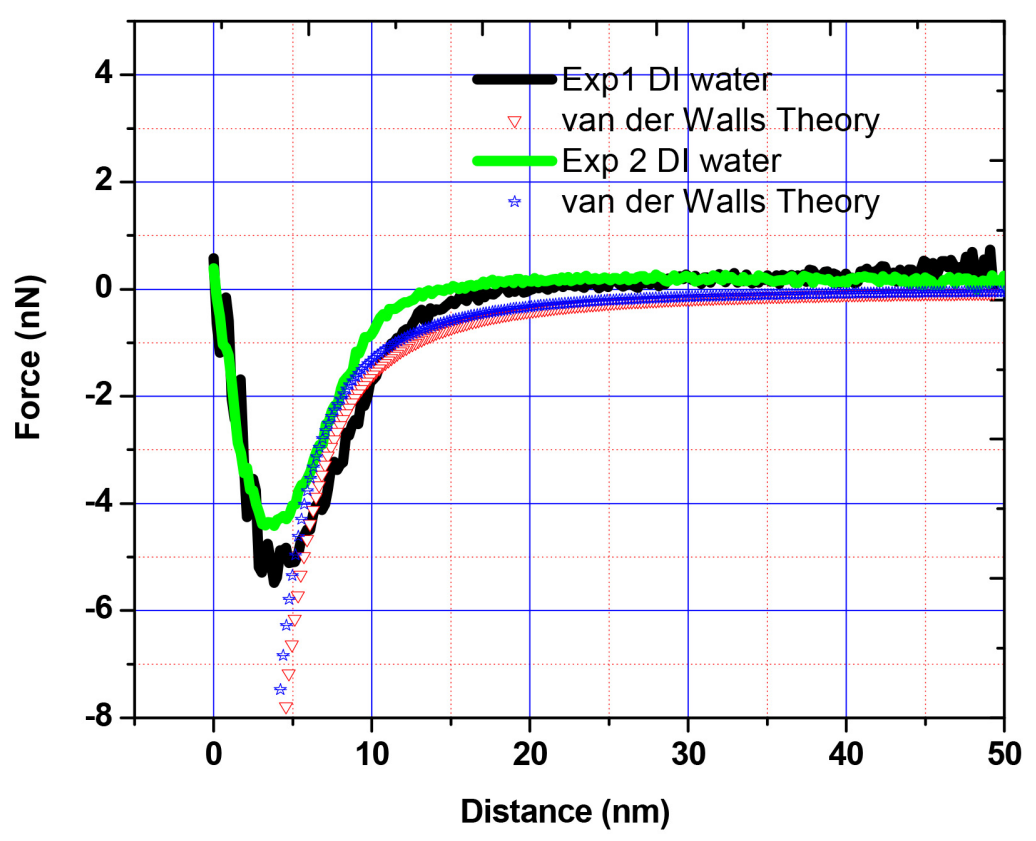

Figure 9. Force versus distance curves in DI water and fit based on the Van der Waals theory.

theory was used. This theory was tested with both a micro and nano colloid probe. All the testing trials matched the theory, though the micro probe's results coincided more with the predicted results for all ranges of force, while the nano probe's test results were good match during the short-ranged forces. This difference in accuracy can be attributed to the formula used for the test theory. In the original model, all calculations were based upon the shape and size of the micro-size probe. Along with measuring surface charge density, this experiment focused on Van der Waals force providing enough information to calculate the Hamaker constant. In an overall conclusion, data analysis from this experiment shows that the obtained surface charge density increases with the ionic concentration. In addition, the nano probes predict higher surface charge density above the silica surface in $\mathrm{KCl}$ solution than the micro-size probe.

\section{Acknowledgements}

We acknowledgement Dr. Shizhi Qian for allowing us to use AFM at Old Dominion University, Norfolk, VA.

\section{Conflicts of Interest}

The authors declare no conflicts of interest regarding the publication of this paper.

\section{References}

[1] Graham, T. (1861) X. Liquid Diffusion Applied to Analysis. Philosophical Transactions of the Royal Society of London, 151, 183-224. 
https://doi.org/10.1098/rstl.1861.0011

[2] Lyklema, J. (2005) Fundamentals of Interface and Colloid Science: Soft Colloids. Vol. 5, Elsevier, Amsterdam, Netherlands.

[3] Tabor, D. and Winterton, R. (1969) The Direct Measurement of Normal and Retarded Van der Waals Forces. Proceedings of the Royal Society of London, Series A, 312, 435-450. https://doi.org/10.1098/rspa.1969.0169

[4] Klein, J. (1983) Forces between Mica Surfaces Bearing Adsorbed Macromolecules in Liquid Media. Journal of the Chemical Society, Faraday Transactions 1: Physical Chemistry in Condensed Phases, 79, 99-118. https://doi.org/10.1039/f19837900099

[5] Parker, J.L., Claesson, P.M., Wang, J.-H. and Yasuda, H.K. (1994) Surface Forces between Plasma Polymer Films. Langmuir, 10, 2766-2773.

https://doi.org/10.1021/la00020a043

[6] Parker, J., Christenson, H. and Ninham, B. (1989) Device for Measuring the Force and Separation between Two Surfaces Down to Molecular Separations. Review of Scientific Instruments, 60, 3135-3138. https://doi.org/10.1063/1.1140544

[7] Claesson, P.M., Ederth, T., Bergeron, V. and Rutlandc, M.W. (1996) Techniques for Measuring Surface Forces. Advances in Colloid and Interface Science, 67, 119-183. https://doi.org/10.1016/0001-8686(96)00302-8

[8] Israelachvili, J. (1991) Intermolecular and Surface Forces. Academic Press, London.

[9] Israelachvili, J. and Berman, A.D. (1999) Surface Forces and Microrheology of Molecularly Thin Liquid Films. In: Bhushan, B., Ed., Handbook of Micro/Nanotribology, 2nd Edition, CRC Press, Boca Raton, Fl.

[10] Israelachvili, J.N. and Adams, G.E. (1978) Measurement of Forces between Two Mica Surfaces in Aqueous Electrolyte Solutions in the Range $0-100 \mathrm{~nm}$. Journal of the Chemical Society, Faraday Transactions 1: Physical Chemistry in Condensed Phases, 74, 975-1001. https://doi.org/10.1039/f19787400975

[11] Larson, I., Drummond, C.J., Chan, D.Y.C. and Grieser, F. (1997) Direct Force Measurements between Silica and Alumina. Langmuir, 13, 2109-2112. https://doi.org/10.1021/la960684h

[12] Parker, J.L. (1994) Surface Force Measurements in Surfactant Systems. Progress in Surface Science, 47, 205-271. https://doi.org/10.1016/0079-6816(94)90019-1

[13] Dorobantu, L.S., Bhattacharjee, S., Foght, J.M. and Gray, M.R. (2009) Analysis of Force Interactions between AFM Tips and Hydrophobic Bacteria Using DLVO Theory. Langmuir, 25, 6968-6976. https://doi.org/10.1021/la9001237

[14] Ducker, W.A., Xu, Z. and Israelachvili, J.N. (1994) Measurements of Hydrophobic and DLVO Forces in Bubble-Surface Interactions in Aqueous Solutions. Langmuir, 10, 3279-3289. https://doi.org/10.1021/la00021a061

[15] Pashley, R. (1981) DLVO and Hydration Forces between Mica Surfaces in $\mathrm{Li}^{+}, \mathrm{Na}^{+}$, $\mathrm{K}^{+}$, and $\mathrm{Cs}^{+}$Electrolyte Solutions: A Correlation of Double-Layer and Hydration Forces with Surface Cation Exchange Properties. Journal of Colloid and Interface Science, 83, 531-546. https://doi.org/10.1016/0021-9797(81)90348-9

[16] Pashley, R. and Israelachvili, J. (1984) DLVO and Hydration Forces between Mica Surfaces in $\mathrm{Mg}^{2+}, \mathrm{Ca}^{2+}, \mathrm{Sr}^{2+}$, and $\mathrm{Ba}^{2+}$ Chloride Solutions. Journal of Colloid and Interface Science, 97, 446-455. https://doi.org/10.1016/0021-9797(84)90316-3

[17] Yoon, R.-H. and Mao, L. (1996) Application of Extended DLVO Theory, IV: Derivation of Flotation Rate Equation from First Principles. Journal of Colloid and Interface Science, 181, 613-626. https://doi.org/10.1006/jcis.1996.0419

[18] Ai, Y., Liu, J., Zhang, B. and Qian, S. (2010) Field Effect Regulation of DNA Trans- 
location through a Nanopore. Analytical Chemistry, 82, 8217-8225. https://doi.org/10.1021/ac101628e

[19] Daiguji, H., Yang, P. and Majumdar, A. (2004) Ion Transport in Nanofluidic Channels. Nano Letters, 4, 137-142. https://doi.org/10.1021/nl0348185

[20] He, Y., et al. (2011) Gate Manipulation of DNA Capture into Nanopores. ACS Nano, 5, 8391-8397. https://doi.org/10.1021/nn203186c

[21] Hsu, C., Lo, T.-W., Lee, D.-J. and Hsu, J.-P. (2013) Electrophoresis of a Charge-Regulated Zwitterionic Particle: Influence of Temperature and Bulk Salt Concentration. Langmuir, 29, 2427-2433. https://doi.org/10.1021/la3047752

[22] Jin, X. and Aluru, N.R. (2011) Gated Transport in Nanofluidic Devices. Microfluidics and Nanofluidics, 11, 297-306. https://doi.org/10.1007/s10404-011-0796-3

[23] Matovic, J., et al. (2012) Field Effect Transistor Based on Ions as Charge Carriers. Sensors and Actuators B: Chemical, 170, 137-142.

https://doi.org/10.1016/j.snb.2011.03.011

[24] Singh, K.P. and Kumar, M. (2012) Effect of Gate Length and Dielectric Thickness on Ion and Fluid Transport in a Fluidic Nanochannel. Lab on a Chip, 12, 1332-1339. https://doi.org/10.1039/c2lc20869e

[25] Vinogradova, O.I., Yakubov, G.E. and Butt, H.-J. (2001) Forces between Polystyrene Surfaces in Water-Electrolyte Solutions: Long-Range Attraction of Two Types? The Journal of Chemical Physics, 114, 8124-8131. https://doi.org/10.1063/1.1365954

[26] Fielden, M.L., Hayes, R.A. and Ralston, J. (1996) Surface and Capillary Forces Affecting Air Bubble-Particle Interactions in Aqueous Electrolyte. Langmuir, 12, 3721-3727. https://doi.org/10.1021/la960145c

[27] Christenson, H.K., et al. (1990) Effect of Divalent Electrolyte on the Hydrophobic Attraction. Journal of Physical Chemistry, 94, 8004-8006. https://doi.org/10.1021/j100384a002

[28] Parker, J.L., Claesson, P.M. and Attard, P. (1994) Bubbles, Cavities, and the Long-Ranged Attraction between Hydrophobic Surfaces. The Journal of Physical Chemistry, 98, 8468-8480. https://doi.org/10.1021/j100085a029

[29] Kokkoli, E. and Zukoski, C.F. (1999) Effect of Solvents on Interactions between Hydrophobic Self-Assembled Monolayers. Journal of Colloid and Interface Science, 209, 60-65. https://doi.org/10.1006/jcis.1998.5862

[30] Zhmud, B., Meurk, A. and Bergström, L. (1998) Evaluation of Surface Ionization Parameters from AFM Data. Journal of Colloid and Interface Science, 207, 332-343. https://doi.org/10.1006/jcis.1998.5783

[31] Weisenhorn, A., Maivald, P., Butt, H.-J. and Hansma, P.K. (1992) Measuring Adhesion, Attraction, and Repulsion between Surfaces in Liquids with an Atomic-Force Microscope. Physical Review B, 45, 11226. https://doi.org/10.1103/PhysRevB.45.11226

[32] Hoh, J.H., Revel, J.-P. and Hansma, P.K. (1991) Tip-Sample Interactions in Atomic Force Microscopy: I. Modulating Adhesion between Silicon Nitride and Glass. Nanotechnology, 2, 119. https://doi.org/10.1088/0957-4484/2/3/003

[33] Hüttl, G., Beyer, D. and Müller, E. (1997) Investigation of Electrical Double Layers on $\mathrm{SiO}_{2}$ Surfaces by Means of Force vs. Distance Measurements. Surface and Interface Analysis, 25, 543-547. https://doi.org/10.1002/(SICI)1096-9918(199706)25:7/8<543::AID-SIA268>3.0.CO; $\underline{2-\mathrm{T}}$

[34] Lin, X.Y., Creuzet, F. and Arribart, H. (1993) Atomic Force Microscopy for Local 
Characterization of Surface Acid-Base Properties. The Journal of Physical Chemistry, 97, 7272-7276. https://doi.org/10.1021/j100130a025

[35] Toikka, G., Hayes, R.A. and Ralston, J. (1996) Adhesion of Iron Oxide to Silica Studied by Atomic Force Microscopy. Journal of Colloid and Interface Science, 180, 329-338. https://doi.org/10.1006/jcis.1996.0311

[36] Rutland, M.W. and Senden, T.J. (1993) Adsorption of the Poly (Oxyethylene) Nonionic Surfactant C12E5 to Silica: A Study Using Atomic Force Microscopy. Langmuir, 9, 412-418. https://doi.org/10.1021/la00026a009

[37] Meagher, L. (1992) Direct Measurement of Forces between Silica Surfaces in Aqueous $\mathrm{CaCl}_{2}$ Solutions Using an Atomic Force Microscope. Journal of Colloid and Interface Science, 152, 293-295. https://doi.org/10.1016/0021-9797(92)90030-P

[38] Cosgrove, T. (2010) Colloid Science: Principles, Methods and Applications. John Wiley \& Sons, Hoboken, NJ.

[39] Israelachvili, J.N. and Tabor, D. (1972) The Measurement of Van der Waals Dispersion Forces in the Range 1.5 to $130 \mathrm{~nm}$. Proceedings of the Royal Society of London. Series $A$, 331, 19-38. https://doi.org/10.1098/rspa.1972.0162

[40] Atalay, S., et al. (2014) Surface Charge of a Nanoparticle Interacting with a Flat Substrate. The Journal of Physical Chemistry C, 118, 10927-10935. https://doi.org/10.1021/jp5023554

[41] Butt, H.-J., Cappella, B. and Kappl, M. (2005) Force Measurements with the Atomic Force Microscope: Technique, Interpretation and Applications. Surface Science Reports, 59, 1-152. https://doi.org/10.1016/j.surfrep.2005.08.003

[42] Hu, K. and Bard, A.J. (1997) Characterization of Adsorption of Sodium Dodecyl Sulfate on Charge-Regulated Substrates by Atomic Force Microscopy Force Measurements. Langmuir, 13, 5418-5425. https://doi.org/10.1021/la970483t 